\title{
Towards Learning Hierarchical Compositional Models in the Presence of Clutter ${ }^{\star}$
}

\author{
Jan Mačák and Ondřej Drbohlav \\ Center for Machine Perception (CMP), \\ Department of Cybernetics, Czech Technical University in Prague \\ \{macakj1,drbohlav\}@cmp.felk.cvut.cz
}

\begin{abstract}
Our goal is to identify hierarchical compositional models from highly cluttered data. The data to learn from are assumed to be imperfect in two respects. Firstly, large portion of the data is coming from background clutter. Secondly, data generated by a recursive compositional model are subject to random replacements of correct descendants by randomly chosen ones at every level of the hierarchy. In this paper, we study the limits and capabilities of an approach which is based on likelihood maximization. The algorithm makes explicit probabilistic assignments of individual data to compositional model and background clutter. It uses these assignments to effectively focus on the data coming from the compositional model and iteratively estimate their compositional structure.
\end{abstract}

\section{Introduction}

A long-term goal in machine learning field is to learn usable models of objects from observations of the world, in an unsupervised way. It is generally believed that suitable representations of the world should be hierarchical and compositional 1110. Such representations are efficient as for the computational resources (due to hierarchy) and in memory requirements (share-ability of parts in compositional structures).

What principles should be used in order to identify structural building blocks in the data? Possibilities are usually centered around the Minimum Description Length (MDL) principle [1, and proceed by looking for what has been termed "suspicious coincidences" in the data (data appearing jointly with probability higher than chance). Notable results have been obtained in specific cases, e.g. in vision (e.g. [14,8] ). Our primary research interest is also centered in vision, and we will be inclined to motivate the design settings of our experiment, or comment on the results, from point of view of a computer vision researcher, while keeping the analysis general. * The authors were supported by the Czech Science Foundation under the Project
P103/12/1578 (SeMoA). 


\subsection{Key Features of This Work}

Our goal is to identify hierarchical compositional models from the observed data. Consider an example of the visual domain: The data would consist of images, and the hierarchical compositional models would comprise of models for shapes and visual qualities of different objects, their parts, and (recursively) the subparts of these parts. In this paper, we however do not wish to be bound to a particular domain (visual, auditory, or other); our aim is to find out whether the proposed algorithm can generally work in such adverse settings. For this reason, we extract two usual aspects of the real-world data which make the task difficult: i) the data are, for a large part, composed of noise, or background clutter; ii) the compositions (or objects) are not present in the data in a clean form, but any part (or its sub-part) in the object's compositional structure may be replaced by a different part. In the visual domain, aspect i) would correspond to the fact that large portions of images are composed of non-object data (textures or uniform areas), while aspect ii) would roughly correspond to occlusion by another object, articulation or changed appearance.

In this paper, we wish to show general capabilities and limits of an approach based on Maximum Likelihood (ML). The aim of our algorithm is to find a suitable set of models (compositions) on each layer of hierarchy such that the data likelihood is maximized (the model is described in a more detail in Section 2). The algorithm works in iterations. In each iteration, it learns the compositions layer by layer, starting from the bottom one, and estimates soft assignments of data to clutter and non-clutter. This enables it to use only the non-clutter data to refine the library of compositions in the next iteration (full description of the algorithm is in Section 3 .)

\subsection{Contribution}

We design an algorithm for compositional models extraction from data which is suited for the two peculiarities of the data. The algorithm proceeds iteratively in a bottom-up and top-down manner. The essential part of the algorithm is that it makes probabilistic assignments of each data to clutter and non-clutter part of the model. We show the capabilities and limits of this ML-based approach on synthetically generated and real data. The study is made on a problem which is simplified to a maximum (aligned data, known graph structure) while maintaining the essential features of the real-world data (high amount of clutter, and missing parts in compositions).

\subsection{Prior Work}

Fukushima [5] was one of the first to research the topic of a neural network able to learn without supervision. His approach has been inspired by biological principles. He constructed a hierarchical neural network and demonstrated its performance on digits recognition. Frey et al. 4] also used a neural network for unsupervised learning and used local rules for adjusting weights on connections 
in it. They were able to adjust weights such that the neural network learns two classes of simple patterns (horizontal and vertical stripes). In effect, they employed the principle of KL divergence minimization. This was followed by the work of Hinton et al. [7] which extended the previous results and proposed efficient algorithm for learning in deep belief nets.

Compositionality has been recently an active topic in the specific area of computer vision. For example, Ommer and Buhmann 9] suggested an algorithm to learn compositional representation of visual objects and used this approach for object recognition. Image patches are used as building blocks for the lowest layer. Leonardis [32] uses sparse features (edges) as building blocks, and constructs a hierarchical model which is, notably, generative. The model is constructed intuitively but not cast in probabilistic framework. Zhu et al. 1413 published works on semi- and unsupervised learning in visual domain combining bottomup and top-down processes. Short theoretical analysis of this problem has been recently performed by Yuille [12].

\section{Model}

A tree-shaped graphical model has been employed in this paper (please see Fig. 1 and the notation symbols explained there) to represent the data. The tree has five layers in total: a root layer consisting of a single node $\sigma$, three inner layers $\mathbf{a}, \mathbf{b}$ and $\mathbf{c}$, and the data layer $\mathbf{D}$. Note that we use the 5-layer model to employ a concrete example but in practice we could use any number of layers.

The root of the graph hosts variable $\sigma$ which can be thought of as representing different objects. Thus its states are from a set $\Sigma$ which represents the set of top-level compositions.

The probability of a set of node values is

$$
P(\sigma, \mathbf{a}, \mathbf{b}, \mathbf{c}, \mathbf{D})=P(\sigma) \prod_{i=1}^{5}\left(P\left(a_{i} \mid \sigma\right) \prod_{j=1}^{5}\left(P\left(b_{i j} \mid a_{i}\right) \prod_{k=1}^{5} P\left(c_{i j k} \mid b_{i j}\right) P\left(D_{i j k} \mid c_{i j k}\right)\right)\right) .
$$

Here, the node names are as described in Fig. 1 (please see this figure for a selfcontained explanation of the graph structure and notation conventions), and the bold-faced letters $\mathbf{a}, \mathbf{b}, \mathbf{c}$ and $\mathbf{D}$ denote a set collecting all the nodes from the respective layers. Note that following (usual) minor abuse of notation, $a_{i}$ is sometimes used both to identify the node in the graph and to denote a specific value $a_{i} \in \mathcal{A}$ in that node.

A state in any of the inner nodes $x$ is either one of the compositions $x \in \mathcal{X}=$ $\left\{\mathrm{x}^{1}, \mathrm{x}^{2}, \ldots, \mathrm{x}^{|\mathcal{X}|}\right\}$, or the background state $\mathrm{x}^{b g}$. Having a parent $x$ and its child $y$ (take e.g. $x=b_{25}$ and $y=c_{251}$, say), the conditional probability $P(y \mid x)$ takes a form according to the type of the state. For compositional states, this probability reflects the preference of the state $\mathrm{x}$ to have a specific part $\mathrm{y}^{k}$ as its child:

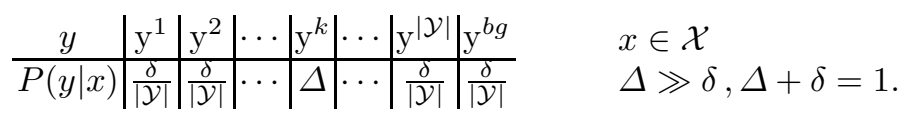




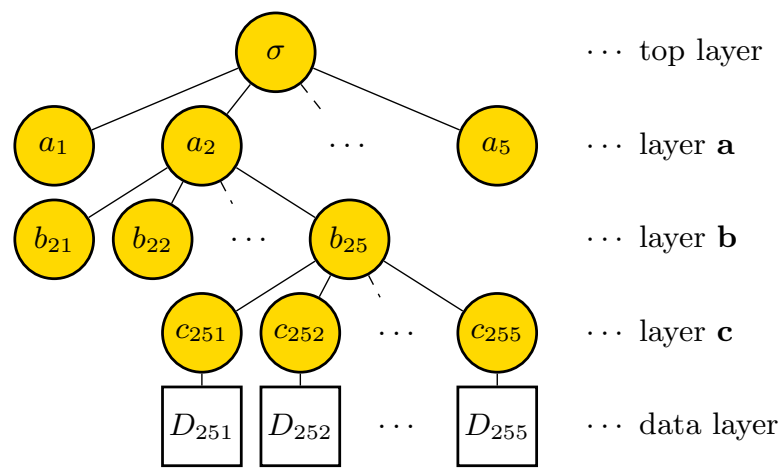

Fig. 1. The structure of the graph. Each node in the root layer and in layer $\mathbf{a}$ and $\mathbf{b}$ has five children, of which only some are shown for the sake of clarity. Nodes in layer c are connected to leaves by single links. The leaves are the data nodes. The nodes are denoted using indexing where children inherit the parent's indices and get one more own index. Thus children of $a_{i}$ are denoted $b_{i j}$ where $j$ identifies one of the five children of $a_{i}$. Likewise, children of $b_{i j}$ are denoted $c_{i j k}$. Compositionality is reflected by that any of the nodes in layer a has states from the same set $\mathcal{A}=\left\{A_{1}, A_{2}, \ldots, A_{|\mathcal{A}|}\right\}$, thus $a_{i} \in \mathcal{A}, \forall i=1,2, \ldots, 5$. The same is true about nodes in layers $\mathbf{b}$ and $\mathbf{c}$. For the same reason of compositionality, the edge potentials for a given parent and child at a given layer are also the same, thus e.g. $\left(b_{i j}=b_{o p} \wedge c_{i j k}=c_{o p k}\right) \Rightarrow P\left(c_{i j k} \mid b_{i j}\right)=P\left(c_{o p k} \mid b_{o p}\right)$.

The other possibility is that $x=\mathrm{x}^{b g}$, and in that case the preference for any of its child's identities is described by the relative frequencies $\left\{\hat{p}_{\mathrm{y}^{1}}, \hat{p}_{\mathrm{y}^{2}}, \ldots, \hat{p}_{\mathrm{y}|\mathcal{Y}|}, \hat{p}_{\mathrm{y}^{b g}}\right\}$ in the data:

$$
\begin{array}{c|c|c|c|c|c|}
y & \mathrm{y}^{1} & \mathrm{y}^{2} & \cdots & \mathrm{y}^{|\mathcal{Y}|} & \mathrm{y}^{b g} \\
\hline P(y \mid x) & \hat{p}_{\mathrm{y}^{1}} & \hat{p}_{\mathrm{y}^{2}} & \cdots & \left|\hat{p}_{\mathrm{y}}\right| \mathcal{Y} \mid & \hat{p}_{\mathrm{y}^{b g}}
\end{array} \quad x=\mathrm{x}^{b g} .
$$

\section{Algorithm}

The task of the algorithm is the following. The input consists of the observed data $D$. The required goal is to find the parameters of the model in the Eq. 1 such that the probability of observing the data is maximized. The parameters of the model are: i) prior probabilities over all layers $(P(\sigma)$ resp. $P(x))$, the lower layer priors are used as $\hat{p}$ in Eq. 3 , and ii) five-tuple of indices $k$ specifying child components, for each $x \in \mathcal{X}$. The conditional probabilities of the data, that is $P\left(D_{i j k} \mid c_{i j k}\right)$, are given.

To learn the model basically means to build up a library of compositions. The library is hierarchically organized and each layer is learned interdependently based on previous layers and has the capability of storing a certain number of compositions. These capacities are set in advance. The learning works in a Bottom-Up manner with Top-Down adjustment. 


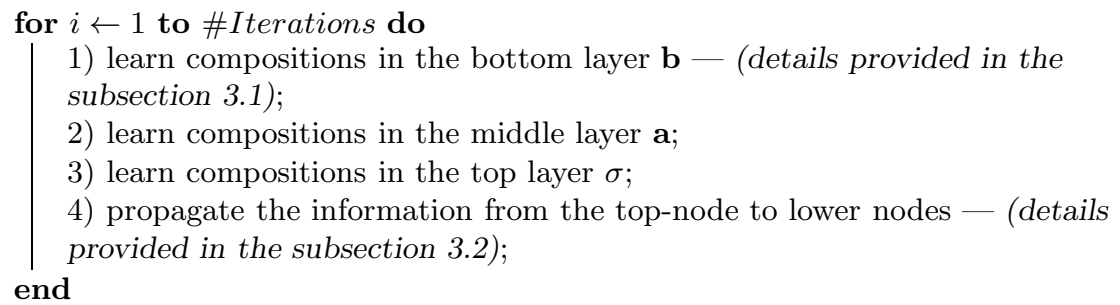

Algorithm 1. Learning algorithm

\subsection{Bottom-Up Learning}

Bottom-up learning is an iterative process performed as the Steps 1)-3) of the Algorithm 1 and deals with learning a single layer. It basically takes the set of all five-tuples in the preceding (lower) layer and comes up with compositions in the current layer which represent them best.

The library is initialized randomly and all compositions are set to be equally likely. The ML estimates for the conditional probabilities $P(y \mid x)$ and prior $P(x)$ are given by

$$
P(y \mid x)=\frac{1}{\lambda_{1}} \sum_{n=1}^{N} P\left(y, x \mid D_{n}\right), \quad P(x)=\frac{1}{\lambda_{2}} \sum_{n=1}^{N} P\left(x \mid D_{n}\right),
$$

provided that we assume for the moment a reduced graphical model only up to the current layer ( $x$ being the top node). In this equation, the summation is over the whole dataset and $\lambda_{1}, \lambda_{2}$ are the normalizing factors. The joint probability is estimated as $P\left(y, x \mid D_{n}\right)=P\left(y \mid D_{n}\right) P\left(x \mid D_{n}\right)$, which means that information from the top node $x$ to the lower-level node $y$ does not affect the marginal at the lower-level node. The conditional probabilities used are estimated using standard Bayes formula from $P\left(D_{n} \mid y\right), P\left(D_{n} \mid x\right)$ and priors $P(x)$ and $P(y)$. The former is estimated simultaneously with the layer compositions, the latter is known from learning of the previous layer.

The probability in Eq. [ is then converted to a composition by taking the max-probability item $\mathrm{y}^{k}$ in the distribution $P(y \mid x)$ and assigning value $\Delta$ to it, while assigning values $\delta /|\mathcal{Y}|$ to all other items. When learning the background (cf. Eq. 3), the conditional probability stays as it is.

The process iterates by re-estimation of assignments $P\left(y \mid D_{n}\right), P\left(x \mid D_{n}\right)$ using adjusted compositions and re-estimation of conditional and prior probabilities. The number of iterations is fixed.

\subsection{Top-Down Adjustment}

Top-down adjustment is performed as the Step 4 of the Algorithm 1. Once the learning process reaches the top layer, it is possible to propagate the top-node information on the soft assignments of individual data vectors to compositional model or to clutter. This essentially helps to focus on non-clutter data in the next algorithm iteration and improve the model. 
The assignment of each data to compositional model or background is propagated by a top-down message constructed as

$$
\begin{array}{c|c|c|c|c|c}
y & \mathrm{y}^{1} & \mathrm{y}^{2} & \ldots & \mathrm{y}^{\mid \mathcal{Y}} \mid & \mathrm{y}^{b g} \\
\hline P(y \mid x), x \in \mathcal{X} & \frac{\Delta}{|\mathcal{Y}|} & \frac{\Delta}{|\mathcal{Y}|} & \ldots & \frac{\Delta}{|\mathcal{Y}|} & \delta \\
P\left(y \mid \mathrm{x}^{b g}\right) & \frac{\delta}{\mathcal{Y}} & \frac{\delta}{\mathcal{Y} \mid} & \ldots & \frac{\delta}{|\mathcal{Y}|} & \Delta .
\end{array}
$$

Consequently, in the next iteration, the non-model data are effectively assigned to the clutter model $\sigma^{b g}$ and do not affect the learning of the compositional model.

Additionally, before propagating the top-down information, models with low significance are removed from library. This is basically a simple implementation of the MDL principle [6]. It means that models which do not contribute much to data log-likelihood are not considered to belong to the model and corresponding data are assigned to be clutter. The contribution of each learned composition is computed as

$$
\Delta L_{\sigma^{i}}=\sum_{n=1}^{N} \ln \frac{P\left(D_{n} \mid \sigma^{i}\right) P\left(\sigma^{i}\right)+P\left(D_{n} \mid \sigma^{b g}\right)\left(1-P\left(\sigma^{i}\right)\right)}{P\left(D_{n} \mid \sigma^{b g}\right)}
$$

where $P\left(\sigma^{i}\right)$ is probability of the compositional model $\sigma^{i}$, the model $\sigma^{b g}$ is the model for clutter. The composition $\sigma^{i}$ is removed if its contribution of the composition is less than threshold value. The Eq. 6] is simply a difference of the baseline data log-likelihood of the model without any compositional model and the data log-likelihood supposing there is a compositional model $\sigma_{i}$ which represents well a $P\left(\sigma^{i}\right)$ portion of data. The values of $P\left(\sigma^{i}\right)$ and $P\left(\sigma^{b g}\right)$ are derived from ML estimates of the distribution of top layer models. Using the value $P\left(\sigma^{i}\right)$ gives a lower bound on $\Delta L_{\sigma^{i}}$, because, under the assumption of no other compositional models, the optimal $P^{*}\left(\sigma^{i}\right)$ must lie within the interval of $\left\langle P\left(\sigma^{i}\right), P\left(\sigma^{b g}\right)\right\rangle$.

In practice, the learning converges in few steps even for the cases of low concentration of a compositional model data in clutter. As can be seen in the Fig. 2, two iterations are sufficient for learning the model.

\section{Experiments}

\subsection{Artificial Data Experiment}

The learning algorithm was tested on artificially generated compositional data. This data was generated from a randomly generated compositional model which consisted of five classes (templates) on the top layer, eight templates on the middle layer and nineteen templates on the bottom layer. Each template was build from five components (templates from the lower layer) randomly. The number of five top classes was chosen small, because the aim was to investigate how the learning worked for highly cluttered data. For high number of classes, the amount of the clutter data would have had to be extremely large. 
The compositional data was generated using the templates converted to potentials specified by Eq. 2. Several values for $\Delta$ were used, of which we show results for $\Delta=0.99$ (lower number of random replacements, thus easier case) and $\Delta=0.75$ (harder case). Background clutter was generated from the uniform distribution over the finite interval of $\langle 1,25\rangle$.

In the learning data, there were 5000 data vectors of which only $5 \%$ was generated by the compositional model. The capacities of library while learning were set to accommodate up to 20,40 and 60 models at the bottom, the middle and the top layer respectively.

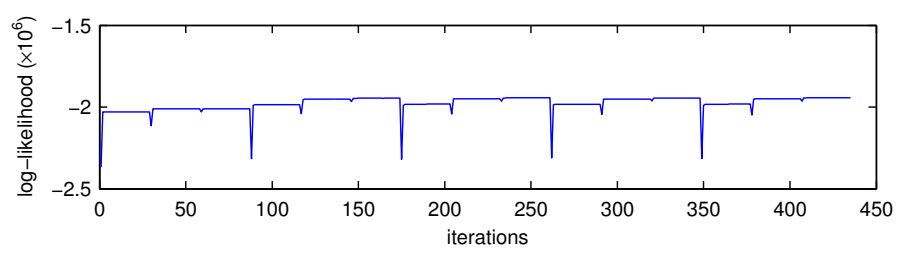

Fig. 2. Development of data log-likelihood. The horizontal axis shows inner cycle iterations. There are 30 iterations at each layer, so 90 iterations are performed in each cycle of Bottom-Up learning. The drop of the log-likelihood after the end of the previous cycle is caused by random initialization of clusters.

The Fig. 3 shows results of learning. It shows the euclidean distance of the learned models and the ground truth. The model was learned correctly as there is zero distance between five models from top layer and generating models. The rate was estimated also correctly, assigning $95 \%$ of data to clutter, and approx. $1 \%$ of data to each of the five models. The true numbers are $95 \%$ clutter, $1 \%$ each compositional model. This result was obtained after five iterations. The assignment of data to individual top-layer models is shown in the Fig. 5 and as can be seen, the actual number of learned models on the top-most layer is correct, despite the fact that the library size is set excessively.

Another interesting question would be that of what is the break-down point of this method. This was estimated experimentally. The experiment was to keep all the statistics and parameters unchanged and iteratively reduce the number of compositional data instances. The total number of data vectors was 1000 and the concentration was falling from $5 \%$ to $0.5 \%$. The algorithm began to fail at around $1.5 \%-2 \%$. Success and failure was measured as whether the data was correctly partitioned.

\subsection{Controlled Experiment with Real Data}

The learning algorithm was tested on a more realistic example of learning an optimal representation for random sequences of characters. The source characters were taken from a scanned letter written on a typewriter, which means that there 


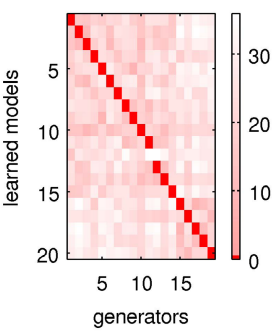

(a)

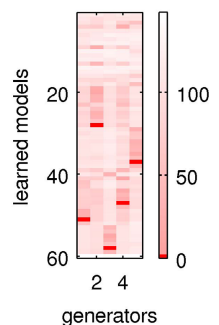

(b)

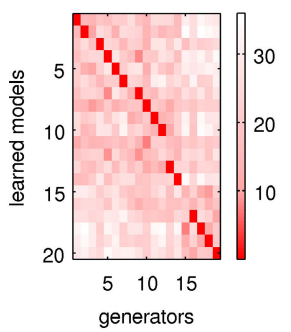

(a)

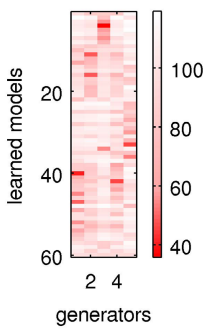

(b)

Fig. 3. Distances of learned models to ground truth, for $\Delta=0.99$. (a) bottom layer $\mathbf{b}$, (b) top layer $\sigma$. Vertical axis shows indices of learned templates, horizontal axis shows indices of ground truth templates. All models have been found.

Fig. 4. Distances of learned models to ground truth, harder data $(\Delta=0.75)$. (a) 1st layer, (b) top layer. It can be seen in the (a) that some compositions were missed (3rd, 8th, 15th).

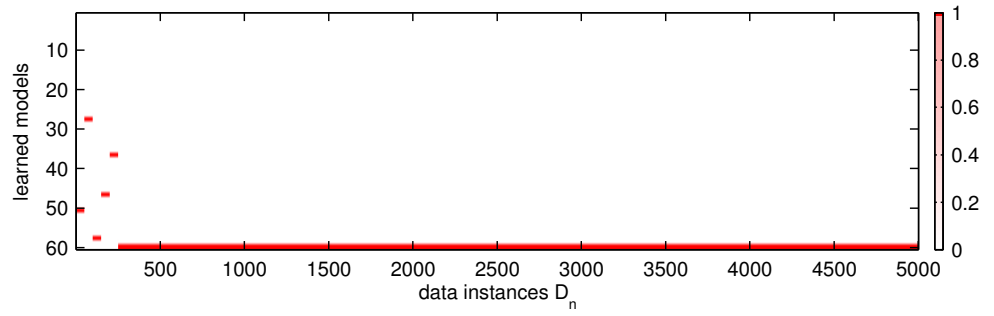

Fig. 5. Top layer model-data assignment. The horizontal axis shows individual data vectors and their assignments to the top layer models (on the vertical axis) the indices correspond to the vertical indices in the Fig. 3(b). The "compositional" data are grouped together and aligned to the left for the sake of visual inspection. The probability of $P\left(\sigma \mid D_{n}\right)$ is color-coded with the scale shown on the right side.

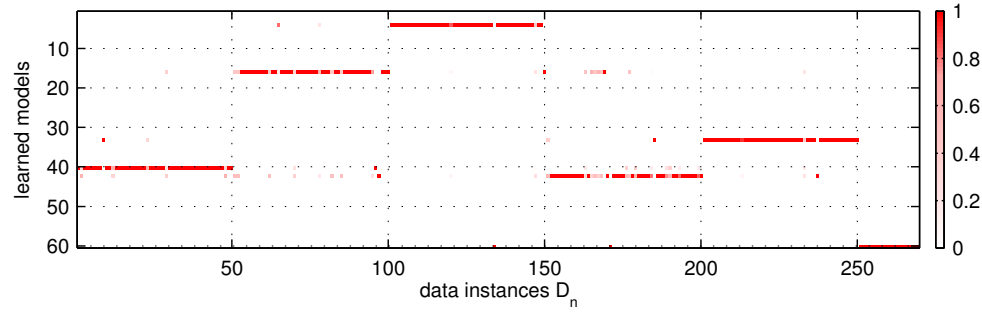

Fig. 6. Top layer model-data assignment for harder data $(\Delta=0.75)$, the meaning is the same as in the Fig. 5] This time, only the detailed picture of the assignment of "compositional" data is shown. It can be seen that majority of data instances are assigned correctly, however there are some wrong assignments. 
are various differences within the sets of same characters. The thickness depends on a stroke style, the noise due to scanning is present, etc.

The data itself was generated in the following way. First, the individual characters were cropped from the picture and coarsely aligned. Then, five random sequences were selected and ten instances for each sequence built using randomly selected character instances. Finally, the data was completed by randomly misaligned samples from the same picture as to obtain the background distribution with similar statistical properties. The ratio between compositional and background data was 1:1 $(5 \times 10$ model instances, 50 random instances $)$. The five sequences to be learned and their learned representations are shown in the Fig. 7 The numbers of elements on individual layers were set to 50, 50 and 10 respectively.

The characters were not learned precisely, but are distinguishable. The interesting observation is that there are both multiple words represented by one model and multiple models for one word. This is caused by the fact that the characters were not aligned precisely and the algorithm is not shift invariant and consequently a slightly shifted letter might get completely different representation on the lowest layer.

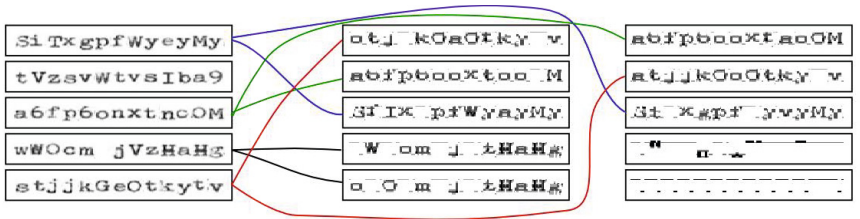

Fig. 7. Ground truth sequences in the left column and their learned representations in the middle and the right column. As can be seen, even for such a small dataset 4 out of 5 sequences were learned.

\section{Conclusion}

Our main goal in this paper was to see how far we can get with likelihood optimization in learning hierarchical models from heavily cluttered data. We have constructed a simple and very controlled framework, and an experiment which enabled us to study the merits of likelihood maximization in isolation.

We have observed that hierarchical models can be learnt even in a very adverse scenario when $95 \%$ of data were outliers. This was greatly helped by the feedback nature of the algorithm, where the bottom-up stage is followed by a top-down step, thus possibly improving the selection of proper models at the lower levels of hierarchy. We have also observed how the results depend on the rate of the clutter data, as well as on the number of random replacements (parameter $\Delta$ ). Experiment on scanned characters confirmed the expected limitation of the algorithm for this type of application due to the requirement of data being aligned with data nodes. 


\section{References}

1. Bienenstock, E., Geman, S., Potter, D.: Compositionality, MDL priors, and object recognition. In: Mozer, M., Jordan, M.I., Petsche, T. (eds.) NIPS, pp. 838-844. MIT Press (1996)

2. Fidler, S., Boben, M., Leonardis, A.: Optimization framework for learning a hierarchical shape vocabulary for object class. In: BMVC. British Machine Vision Association (2009)

3. Fidler, S., Leonardis, A.: Towards scalable representations of object categories: Learning a hierarchy of parts. In: Proc. CVPR (2007)

4. Frey, B., Dayan, P., Hinton, G.E., Jenkin, I.M.: A simple algorithm that discovers efficient perceptual codes. In: Mechanisms, L.R.H., Mechanisms, B. (ed.) Computational and Psychophysical Mechanisms of Visual Coding, pp. 296-315. Cambridge University Press (1997)

5. Fukushima, K.: Neocognitron: A hierarchical neural network capable of visual pattern recognition. Neural Networks 1(2), 119-130 (1988), http://www.sciencedirect.com/science/article/pii/0893608088900147

6. Grünwald, P.D.: The Minimum Description Length Principle. MIT Press (2007)

7. Hinton, G.E., Osindero, S.: A fast learning algorithm for deep belief nets. Neural Computation 18 (2006)

8. Kokkinos, I., Yuille, A.: Inference and learning with hierarchical shape models. International Journal of Computer Vision 93, 201-225 (2011), http://dx.doi.org/10.1007/s11263-010-0398-7

9. Ommer, B., Buhmann, J.: A compositionality architecture for perceptual feature grouping. In: Rangarajan, A., Figueiredo, M.A.T., Zerubia, J. (eds.) EMMCVPR 2003. LNCS, vol. 2683, pp. 275-290. Springer, Heidelberg (2003)

10. Ommer, B., Buhmann, J.: Learning the compositional nature of visual object categories for recognition. IEEE Trans. Pattern Anal. Mach. Intell. 32(3), 501-516 (2010)

11. Tsotsos, J.K.: Analyzing vision at the complexity level. Behavioral and Brain Sciences 13(03), 423-445 (1990), http://dx.doi.org/10.1017/S0140525X00079577

12. Yuille, A.L.: Towards a theory of compositional learning and encoding of objects. In: ICCV Workshops, pp. 1448-1455. IEEE (2011)

13. Zhu, L., Chen, Y., Yuille, A.L.: Learning a hierarchical deformable template for rapid deformable object parsing. IEEE Trans. Pattern Anal. Mach. Intell. 32(6), 1029-1043 (2010)

14. Zhu, L., Chen, Y., Yuille, A.L.: Recursive compositional models for vision: Description and review of recent work. Journal of Mathematical Imaging and Vision 41(12), 122-146 (2011) 